\title{
ESSAI DE RADIO-PISTAGE SUR LA CARPE
}

\section{P. STEINBACH *}

* Ingénieur I.S.I.M., Conseil Supérieur de la Pêche - 112, Faubourg de la Cueille - 86000 POITIERS, France..

\section{RESUME}

Deux carpes adultes (Cyprinus carpio) sont équipées d'émetteurs radio et relâchées en Vienne sur leur lieu de capture. La première est marquée par implantation abdominale et la seconde par voie buccale. Les carpes apparaissent peu traumatisées au vu des déplacements qu'elles effectuent. Leur pistage sur sept semaines montre que chacune des deux techniques de marquage peut donner des résultats satisfaisants sur ce poisson.

\section{SUMMARY}

\section{RADIO-TRACKING TEST ON CARP}

Two adult carps (Cyprinus carpio) were tagged with radio transmitters and released in the River Vienne where they were caught. One of them was tagged by mounting in the abdominal cavity and the other one via the mouth. Carp kept on having high moving ability and did not appear affected by handling stress. After seven weeks tracking it appeared that both tag mounting - gut and abdominal - can be successful in carp.

\section{INTRODUCTION}

Les deux communications, "Radio-pistage de saumons adultes en Loire" (BARIL et GUENEAU, 1986) et "Radio-pistage de grandes aloses adultes en Loire" (STEINBACH et al., 1986) portent essentiellement sur l'étude de grands migrateurs et préconisent l'utilisation d'une technique bien adaptée aux comportements natatoires et alimentaires de ces poissons (nage peu profonde, alimentation suspendue pendant la migration).

Par extension, on teste ici le même outil d'investigation sur une espèce différente : la carpe, poisson sédentaire qui évolue en eau plus profonde. Cet essai nous amène conjointement à aborder un dernier type de marquage : l'implantation chirurgicale de l'émetteur dans la cavité générale du poisson.

Les moyens mis en cuvre se limitant à deux émetteurs, les résultats seront principalement commentés d'un point de vue méthodologique.

\section{- Site d'étude :}

Il s'agit d'un tronçon de rivière situé sur la Vienne en aval de CHAUVIGNY (VIENNE). Délimité par deux seuils de moulins désaffectés, ce tronçon présente en moyenne sur ses $3,2 \mathrm{~km}$ une largeur de $70 \mathrm{~m}$ et une profondeur de $3 \mathrm{~m}$. Un radier marqué le divise en deux biefs (1/3 pour le bief amont et $2 / 3$ pour le bief aval).

On distingue sur $200 \mathrm{~m}$ en aval de ce radier le secteur de pêche à l'intérieur duquel les carpes ont été capturées et relâchées une fois marquées. Outre la proximité du radier, ce secteur est caractérisé par un profond de 5 à $6 \mathrm{~m}$.

\section{I - MATERIEL ET METHODES}

\section{- Matériel radiotélémétrique}

Les émetteurs sont ceux utilisés pour le radio-pistage des aloses (STEINBACH et al., 1986). De même, la réception est assurée par des récepteurs portables monocanaux munis d'antennes boucles. La combinaison de ces différents éléments permet d'atteindre des portées de 500 à $600 \mathrm{~m}$ lorsque l'émetteur se trouve immergé sous $1 \mathrm{~m}$ d'eau. Par contre, à $6 \mathrm{~m}$ de profondeur, la distance maximale de réception n'excède pas $65 \mathrm{~m}$.

\section{- Capture et marquage}

Durant la première semaine du mois de septembre 1985, deux carpes capturées à la ligne par un pêcheur amateur sont transportées et placées quelques jours en bac vivier, dans l'attente du marquage. 


\begin{tabular}{|c|c|c|c|c|}
\hline & Variété & $\begin{array}{l}\text { Longueur } \\
\text { totale }(\mathrm{mm})\end{array}$ & Poids (g) & $\begin{array}{l}\text { Durée de vie } \\
\text { de l'émetteur }\end{array}$ \\
\hline Carpe $n^{0} \quad l$ & Carpe miroir & 640 & 5000 & 50 jours \\
\hline Carpe $n^{\mathbf{0}} 2$ & Carpe commune & 600 & 3300 & 44 jours \\
\hline
\end{tabular}

Tableau 1 : Caractéristiques des poissons marqués et des émetteurs.

Table 1 : Characteristics of tagged fishes and transmitters.

Le choix du marquage externe (émetteur fixé à la base de la nageoire dorsale) n'est pas retenu en raison des risques d'arrachage auxquels est exposé l'appareil. Une carpe équipée selon cette technique chercherait par différents moyens à se débarasser de sa charge (TROCHERIE et BERCY, 1984).

1) Le marquage du sujet no 1 est réalisé par voie chirurgicale. L'individu est anesthésié (MS $222-100 \mathrm{mg} / \mathrm{l}$ ) puis sa paroi est incisée longitudinalement sur $2,5 \mathrm{~cm}$, en arrière de la ceinture pelvienne. Le corps de l'émetteur est ensuite délicatement inséré en biais à l'intérieur de la cavité abdominale, en présentant son extrémité sans antenne vers la queue du poisson. Dans le cas présent, on choisit de laisser sortir le fil d'antenne à l'extérieur de l'abdomen, car son insertion derrière l'épaisseur des chairs affecte inévitablement la portée du signal émis. Enfin, l'incision est recousue bord a bord grâce à cinq points de suture au moyen de fil non résorbable (Mersuture 2.0, aiguille courbe).

Remarque : Cette opération est plus facile à réaliser sur des carpes miroir ou cuir de par leur absence partielle ou totale d'écailles.

2) La carpe no 2 est donc, comme les saumons ou les aloses, équipée par voie buccale au moyen d'un jeu de deux tubes (BARIL et GUENEAU, 1986). Afin de la ménager pendant la manipulation et de bloquer son réflexe de régurgitation, celle-ci est préalablement anesthésiée. De plus, pour une meilleure rétention de l'émetteur, l'appareil est poussé profondément (sur environ $10 \mathrm{~cm}$ ) à l'intérieur du tube digestif de la carpe, rectiligne dans sa partie antérieure et dépourvu d'estomac. Une fois l'opération terminée, au plus quelques centimètres du fil d'antenne dépassent de la bouche du poisson.

\section{- Pistage}

Chacune des carpes est repérée au moins une fois par jour. Les localisations sont réalisées, soit par multiples pointages directionnels le long de la berge, soit par prospection fluviale depuis une embarcation non motorisée. Ce dernier mode de repérage est plus précis que le précédent, il permet de situer un poisson immobile dans un rayon de $2 \mathrm{~m}$.

Les conditions de débit rencontrées étant particulièrement stables (étiage exceptionnel), la température, relevée quotidiennement, est le seul facteur contrôlé au cours des opérations de pistage.

\section{II - RÉsultats - Discussion}

\section{- Température}

Durant les 28 premiers jours de suivi la température se maintenant au voisinage de $20^{\circ} \mathrm{C}$ a été stable et favorable a l'activité de la carpe. Ensuite, les eaux se sont progressivement refroidies pour atteindre $10^{\circ} \mathrm{C}$ a la date d'extinction du dernier émetteur.

\section{- Pistage}

Pendant les premières semaines consécutives à leur lâcher, les carpes se sont maintenues à l'intérieur du secteur de pêche, s'éloignant peu de sa zone la plus profonde. Après cette première phase de comportement (15 jours pour la carpe $n^{\circ} 2$ et 32 jours pour la carpe $n^{\circ} 1$ ) les deux individus ont effectué un mouvement anadrome significatif franchissant le radier pour venir évoluer à l'intérieur du bief amont du site. Au vu de l'effort de nage que nécessite pour la carpe ce mouvement anadrome, il s'agirait d'une réponse à certains facteurs trophiques plutôt que l'expression d'un comportement erratique. Enfin, peu de temps avant l'extinction de leurs émetteurs, les deux poissons se sont immobilisés, vraisemblablement conditionnés par le refroidissement progressif des eaux. Le sujet $n^{\circ} 1$ 
s'est immobilisé en premier. La température de l'eau était alors descendue au-dessous de $15^{\circ} \mathrm{C}$. Le sujet $n^{\circ} 2$, plus actif que son congénère a eu le même comportement 6 jours plus tard à un niveau de température légèrement inférieur $\left(13^{\circ} \mathrm{C}\right.$ environ).

II est à noter que malgré des conditions géographiques favorables au pistage (site barré en amont et en aval, largeur mouillée inférieure a $100 \mathrm{~m}$ ), et l'emploi d'un matériel éprouvé sur un fleuve dont les dimensions ne sont pas comparables, les repérages ont parfois été délicats en raison de la profondeur des zones de prédilection de la carpe. La surface d'écoute permettant de couvrir la rivière d'une rive à l'autre avec une marge de sécurité réduite près des fosses, le recours aux prospections fluviales s'est révélé être la solution la plus sûre pour les opérations de repérage quotidien.

\section{- Fiabilite des procédures de marquage}

1) Plusieurs semaines après leur manipulation, les deux poissons ont fait preuve d'une excellente capacité de déplacements actifs, notamment à travers leur effort de franchissement. Ce résultat positif présente un intérêt particulier dans le cadre de l'essai d'implantation chirurgicale. En effet, on pouvait craindre pour la carpe $n^{\circ} 1$ certaines complications physiologiques immédiates ou d'importantes perturbations comportementales consécutives au stress de l'opération.

Remarque : si les portées du matériel radiotélémétrique le permettent, il est bien sûr préférable de glisser l'antenne d'émission à l'intérieur de la cavité abdominale du poisson car son flottement à l'extérieur des chairs constitue une source d'infection potentielle supplémentaire.

2) Comparée à l'implantation chirurgicale, l'insertion par voie buccale est plus simple à réaliser et moins traumatisante pour le sujet manipulé. En revanche, la rétention définitive de l'émetteur à l'intérieur du poisson n'est pas assurée. Les risques de régurgitation seraient cependant minimes chez la carpe : en effet, d'après une dissection réalisée sur un individu marqué gardé en vivier et d'après les bons résultats obtenus sur le sujet n 2 , l'émetteur une fois placé dans la partie antérieure de l'intestin aurait tendance à être ingéré encore plus profondément.

Si l'insertion par voie buccale apparaît techniquement réalisable chez la carpe, notons qu'elle peut fausser les observations faites dans le cadre de suivis prolongés, soit par une modification du comportement alimentaire, soit par une complication intestinale due à l'occlusion engendrée.

\section{III - CONCLUSION}

En matière de radio-pistage, un des choix techniques les plus importants réside dans la sélection d'un mode de marquage adapté à l'espèce étudiée et aux conditions d'expérimentation rencontrées. En ce qui concerne la carpe, les deux solutions ici testées s'avèrent techniquement réalisables. D'une part, ce poisson résistant semble supporter l'implantation chirurgicale sans manifester de trouble apparent de comportement, d'autre part, ses risques de régurgitation apparaissent limités en cas d'insertion par voie buccale.

Le choix de l'une ou l'autre technique dépend donc du champ d'application de chacune :

- l'insertion par voie buccale se prête plutôt à des pistages de courtes durées ou à des observations indépendantes du comportement alimentaire (réactions aux écarts de températures, comportement natatoire, suivis en période de croissance ralentie, etc.) :

- l'implantation abdominale présente peu de limite d'utilisation si ce n'est une éventuelle contreindication en période de frai (affaiblissement physiologique, encombrement de la cavité générale).

Grâce à ce dernier type de marquage et à l'emploi d'émetteurs performants, on peut envisager la réalisation de suivis sur une année complète. De telles possibilités d'investigation présentent bien sûr un intérêt particulier dans le cadre de recherches sur la biologie et l'écoéthologie de la carpe. La principale limitation technique rencontrée réside plus dans la propagation du signal sous l'eau que dans le marquage lui-même. Si le matériel radio est particulièrement fiable pour suivre des grands migrateurs tel que le saumon ou la grande alose en eau douce, le choix d'appareils ultrasonores peut être préférable pour un poisson sédentaire d'eau profonde tel que la carpe.

\section{BIBLIOGRAPHIE}

TROCHERIE F., BERCY C., 1984. Marquage du Brochet (Esox lucius L.) et de la Carpe (Cyprinus carpio L.) dans la Seine au niveau de Montereau, à l'aide d'émetteurs ultrasonores. Cahier Lab. Montereau, $15: 13-20$. 


\section{REMERCIEMENTS :}

De nombreux organismes et de nombreuses personnes nous ont apporté un précieux concours. Nous tenons notamment à remercier :

- G. ANDRE, Garde-Pêche, C.S.P.

- D. AUDIGUE, pêcheur amateur

- A. AUTUORO, Garde-Chef, C.S.P.

- J.P. BARILLOT, Garde-Pêche, C.S.P.

- D. BROUSSARD, Garde-Pêche, C.S.P.

- C. BURGER, Fish and Wildlife Service (U.S.)

- V. COT, Ingénieur stagiaire

- Electricité de France: Région d'Equipement de Tours, Centre de Production Nucléaire de Saint-Laurent-des-Eaux, de Dampierre-en-Burly, de Belleville-sur-Loire.

- Fédérations Départementales des A.A.P.P. du Loir-et-Cher et de la Vienne

- M.C. HILAIRE, Chargée d'Etude

- M. METZ, Ingénieur stagiaire

- M. MILLON, Garde-chef Principal, C.S.P.

- Y. RABIN, pêcheur professionnel

- M. RIGAUDEAU, Garde-Pêche, C.S.P.

- E. SIEGLER, pêcheur professionnel. 